\title{
Evolución de las estrategias de planificación y revisión textual y su relación con el producto escrito
}

\section{Evolution of textual planning and revision strategies and their relationship with the written product}

DOI: http://dx.doi.org/10.17981/cultedusoc.12.2.2021.01

Recibido: 5 de enero de 2021 Aceptado: 7 de mayo de 2021 Publicado: 1 de julio de 2021.

\author{
Patricia Robledo-Ramón \\ Universidad de León. León (España) \\ probr@unileon.es
}

Vanesa García-Gutiérrez

Universidad de León. León (España)

vgarcg02@estudiantes.unileon.es

Para citar este artículo:

Robledo-Ramón, P. y García-Gutiérrez, V. (2021). Evolución de las estrategias de planificación y revisión textual y su relación con el producto escrito. Cultura, Educación y Sociedad, 12(2), 9-26. DOI: http://dx.doi.org/10.17981/cultedusoc.12.2.2021.01

\section{Resumen}

La escritura es fundamental en el ámbito escolar, siendo necesario conocer el proceso de adquisición de las habilidades de planificación y revisión textual que posibilitan su dominio. En este artículo se analizan comparativamente las habilidades y estrategias de escritura relativas a proceso de planificación y revisión textual que presentan estudiantes de $4^{\circ}, 5^{\circ}$ y $6^{\circ}$ curso de Educación Primaria, así como la calidad de sus productos escritos, con la finalidad de identificar el patrón evolutivo de la adquisición de las habilidades cognitivas superiores implicadas en la competencia escrita y su posible relación con el producto escrito. El estudio se basa en un diseño de enfoque cuantitativo y carácter comparativo llevado a cabo con una muestra de 289 estudiantes (9-12 años), escolarizados en un mismo centro educativo en la provincia de León, España. Los estudiantes debieron elaborar varios textos escritos, evaluados con base a diferentes medidas de estructura, coherencia y calidad del producto textual. Los resultados destacan que la planificación textual es mínima, si bien los educandos de $6^{\circ}$ son los que más ideas generan en sus borradores. En relación a la revisión, los alumnos menores son los que detectan más errores mecánicos. No hay diferencias en la detección de errores sustantivos ni en el tipo de estrategias de corrección de errores mecánicos desplegadas, pero sí en las activadas para corregir errores sustantivos. Como conclusión se evidencia una maduración mínima pero progresiva de las habilidades de planificación y revisión textual de los alumnos a medida que aumentan de curso, así como una mejoría en sus productos escritos.

Palabras clave: Composición escrita; planificación textual; detección de errores; estrategias de corrección; calidad textual; estudio evolutivo

\section{Abstract}

Writing is essential in the school environment, being necessary to know the process of acquiring the planning and textual revision skills that allow its mastery. This article comparatively analyzes the writing skills and strategies related to the planning process and textual revision presented by students of 4 th, 5 th and 6 th grade of Primary Education, as well as the quality of their written products, in order to identify the pattern development of the acquisition of higher cognitive skills involved in written competence and its possible relationship with the written product. The study is based on a design with a quantitative approach and comparative character carried out with a sample of 289 students (9-12 years old), enrolled in the same educational center in the province of León, Spain. The students had to elaborate several written texts, evaluated based on different measures of structure, coherence and quality of the textual product. The results highlight that textual planning is minimal, although 6th grade students are the ones that generate the most ideas in their drafts. In relation to the revision, the youngest students are the ones who detect more mechanical errors. There are no differences in the detection of substantive errors or in the type of mechanical error correction strategies deployed, but there are differences in those activated to correct substantive errors. As a conclusion, there is evidence of a minimal but progressive maturation of the students' planning and textual revision skills as they increase the course, as well as an improvement in their written products.

Keywords: Written composition; text planning; error detection; correction strategies; textual quality; evolutionary study 


\section{INTRODUCCIÓN}

\section{Contextualización}

En el sistema educativo actual, la competencia lingüística es una de las más relevantes, debido a su importancia para el desarrollo del niño en sociedad (García, 2015). Tanto es así, que el sistema educativo español (contexto en el que se integra la presente investigación), refleja en su actual Ley Orgánica 8/2013, para la Mejora de la Calidad Educativa (LOMCE, 2013), la importancia de desarrollar la competencia lingüística en los centros educativos. La competencia lingüística abarca desde el componente pragmático-discursivo, que incluye la dimensión sociolingüística vinculada a la producción de mensajes, hasta el componente estratégico, que incluye la adquisición de destrezas y estrategias comunicativas, como es la escritura (Vásquez-Bustamante, 2018). El Real Decreto 126/2014, por el cual se establece el currículo básico de la Educación Primaria Obligatoria (EPO, 2014), recoge en el área de Lengua Castellana y Literatura, la necesidad de instruir a los alumnos en la enseñanza de los procesos de escritura para lograr que las producciones escritas del alumnado se estructuren en tres partes: planificación del escrito, redacción a partir de borradores y revisión de estos antes de redactar el texto definitivo. Así pues, teniendo en cuenta que la composición de textos es una tarea compleja cuyo dominio demanda el conocimiento y la activación adecuada de habilidades de planificación y revisión de textos, además del control automatizado de las habilidades mecánicas o de bajo nivel cognitivo, referidas a la ortografía, gramática o caligrafía (Graham, 2018a, 2018b; Hayes, 1996), es necesario conocer el potencial de aprendizaje que tienen los alumnos en relación a la adquisición de habilidades de planificación y revisión textual.

La planificación consiste en definir los objetivos y elaborar el guión que se seguirá a la hora de elaborar el texto, activándose los subprocesos de generación y organización de ideas (Hayes \& Flower, 1980). La planificación se puede producir en dos momentos: antes o durante la elaboración del texto, pudiendo diferenciar así entre la planificación avanzada, que se desarrolla antes de elaborar el texto y consiste en elaborar un borrador en el que se organizan las ideas; y la planificación en línea, que se desarrolla durante la escritura y consiste en organizar la información a medida que se elabora el texto (Berninger y Swanson, 1994; Limpo, Alves \& Fidalgo, 2014). El tipo de planificación que se emplea ante una tarea de escritura, permite categorizar al escritor en escritor experto o escritor novato. Según esta clasificación, un escritor novato sería aquel que no planifica sus textos o que únicamente escriben las ideas pero no atienden a aspectos como la audiencia, el tema, el objetivo, la coherencia o la cohesión del texto. Por el contrario, se entiende por escritor experto aquel que elabora un borrador y lo revisa para dotarlo de coherencia y estructura dando como resultado un texto final de calidad (Hurtado, 2019; Torrance, Thomas \& Robinson, 1999).

Según Limpo et al. (2014), los niños de siete años ya tienen desarrollada la planificación en línea y no es hasta los once cuando desarrollan la planificación avanzada. De modo que los procesos de planificación sufren una evolución progresiva demostrado que cuanto más pequeño es el niño escritor, más dificultades presentará a la hora de planificar un texto y generar ideas de forma que el borrador elaborado durante la planificación no se 
diferenciará del texto final y se utilizará únicamente para escribir un texto muy similar. Será a los diez años cuando los escritores activen la memoria a largo plazo ante una tarea de escritura, y a los catorce años cuando el proceso de planificación textual consista en apuntar ideas o notas que después serán transcritas al texto final (Rodríguez y Gallego, 2017; Vieiro, 2007).

Por su parte, la revisión consiste en leer el texto y hacer las correcciones necesarias para lograr un texto final de calidad. En este proceso intervienen los subprocesos de lectura y detección de errores y el subproceso de edición y corrección de los mismos (Hayes \& Flower, 1980; García y Arias-Gundín, 2004). Centrándonos en este último subproceso, se distinguen diferentes estrategias de revisión en función de los cambios que se realicen para rectificar los errores que hayan sido detectados, destacando así las estrategias de añadir, quitar, cambiar o reorganizar contenido (Arias-Gundín y García, 2007; Rodríguez y Gallego, 2017).

Al igual que la planificación, la revisión del texto se puede producir en diferentes momentos, distinguiendo así entre la revisión en línea, producida durante el proceso de escritura o inmediatamente después de finalizar el texto, y que se centra especialmente en los aspectos de tipo mecánico; y la revisión posterior a la escritura (Arias-Gundín y García-Sánchez, 2006; Berninger y Swanson, 1994), durante la cual se revisa el texto en mayor profundidad y se realizan revisiones tanto mecánicas como sustantivas (Chanquoy, 2001). La revisión puede atender, por lo tanto a diferentes niveles distinguiendo así entre una revisión mecánica o superficial, cuyas correcciones se centran en la ortográfica, la gramática y la presentación; y una revisión sustantiva o profunda, focalizada en los aspectos sustantivos que afectan al significado de la frase o texto porque hacen referencia a su estructura, coherencia y significado (Arias-Gundín y García, 2007, González, 2020; Turgut, 2019).

La evolución de los procesos de revisión depende de la experiencia como escritor, y se diferencia una vez más, entre escritores novatos y escritores expertos. Los primeros serán aquellos cuya revisión del texto apenas influye en la calidad final del mismo, ya que se centrarán sobre todo en aspectos mecánicos y estéticos del texto; mientras que los escritores expertos se preocuparan por revisar el contenido y la estructura del texto para que el texto sea lo más claro y ordenado posible (Álvarez-Fernández y García-Sánchez, 2014; Rodríguez y Gallego, 2017). El estudio de Limpo et al. (2014) refleja que los niños de entre nueve y doce años tienen una mayor dificultad para detectar los errores de significado, debido, probablemente a que carecen de estrategias de detección o corrección, o a que las revisiones de tipo sustantivo requieran mayor esfuerzo para acceder a la memoria de trabajo.

Diversos estudios han comprobado la importancia que tiene la escritura en la educación, especialmente al comprobar que el rendimiento académico se relaciona estrechamente con la adquisición de la competencia escrita (Sarmiento y Ojeda, 2018; Victoria, EsquivelAncona y Aldrete-Cortez, 2019). Pese a ello y a la gran importancia que los sistemas educativos otorgan a los procesos de composición escrita, existen pocos estudios en el contexto hispano destinados a la evaluación de los procesos de planificación y la revisión textual implicados en la competencia escrita ya detallar cómo evolucionan y se integran estos procesos en la enseñanza primaria (Herrera, 2020). Por otro lado, la legislación 
educativa, en el caso concreto de España, contexto en el que se integra esta investigación, establece que en esta etapa deben trabajarse los procesos cognitivos de escritura, pero no se concreta en qué cursos se deben instruir, por lo que habría que identificar la competencia de los alumnos en cada curso para, a partir de ello, ofrecer procedimientos instruccionales acordes a las capacidades y necesidades del alumnado de las diferentes edades y cursos. De este modo, dada la importancia que tiene la competencia lingüística en el sistema educativo y la sociedad, se presenta un estudio que pretende contribuir a aportar conocimientos científicos sobre la composición escrita en el contexto escolar, centrándose en conocer cómo varían los procesos de escritura de alto nivel cognitivo en alumnos de los últimos cursos de Primaria.

\section{Objetivos e hipótesis}

El objetivo de este articulo es analizar comparativamente las habilidades y estrategias de escritura o composición escrita relativas al proceso de planificación textual (generación de ideas y tipo de planificación) y al de revisión textual (detección y corrección de errores) que presentan el alumnado de $4^{\circ}, 5^{\circ}$ y $6^{\circ}$ curso de Educación Primaria, así como la calidad de sus productos escritos, todo ello con la finalidad de identificar el patrón de adquisición evolutiva de las habilidades cognitivas superiores implicadas en la competencia escrita y su posible relación con el producto escrito.

Tras el análisis de diversos estudios, se parte de la hipótesis de que cuanto más cerca se esté del final de la etapa de Educación Primaria, más desarrolladas estarán las habilidades de planificación y revisión textual de los estudiantes. Esta hipótesis se fundamenta en estudios que demuestran cómo los alumnos de menor edad planifican y revisan sus textos en menor medida o mediante estrategias más sencillas y menos maduras que el alumnado de cursos superiores (Álvarez-Fernández y García-Sánchez, 2014; Limpo et al., 2014). Además, en cuanto a la revisión, es esperable que se centre fundamentalmente en detectar errores mecánicos, si bien se prevé un despliegue diferente de estrategias diversas de corrección en función del curso, variando entre estrategias tales como añadir, quitar, cambiar y reordenar (Arias-Gundín y García, 2008). Se predice un impacto positivo entre la activación de estrategias de planificación y revisión textual y la calidad del producto escrito (Robledo-Ramón \& García-Sánchez, 2013; Aguirre y Negri, 2019), de este modo se espera que los alumnos mayores sean quienes realicen mejores producciones textuales, al suponerse un mayor desarrollo de sus habilidades planificadoras y revisoras.

\section{Metodología}

\section{Muestra}

La muestra que compone el estudio fue seleccionada a través de un proceso de muestreo no probabilístico por conveniencia, y pertenece a un centro educativo de línea cuatro (es decir, cuatro grupos-clase en cada curso) ubicado en España. La muestra inicial estaba compuesta por 294 estudiantes pero tras excluir alumnos que no realizaron todas las sesiones de evaluación, o que, por tener algún diagnóstico de dificultades de aprendizaje o necesidades educativas especiales, no elaboraron textos válidos para el estudio, la muestra 
total la formaron un total de 289 alumnos. Estos cursaban el segundo internivel de E.P.O. (de $4^{\circ}$ a $6^{\circ}$ curso), siendo 91 los alumnos de $4^{\circ}, 99$ alumnos de $5^{\circ}$ y 99 alumnos de $6^{\circ}$ curso, teniendo por lo tanto, edades comprendidas entre los 9 y los 12 años. La muestra estaba compuesta por un $45 \%$ de mujeres y un $55 \%$ de varones.

\section{Instrumentos}

Para la evaluación del producto textual y del proceso de planificación, los alumnos elaboraron dos textos, uno narrativo y otro argumentativo de comparación y contraste. Para su redacción se les proporcionó una hoja para elaborar un borrador, y otra para el propio texto.

Los datos del proceso de planificación se obtuvieron a través del análisis de las hojas de borrador. Para ello se evaluó, por una parte, la productividad o generación de ideas, contabilizando el número de palabras escritas en el borrador, y por otra parte el tipo de planificación realizada por el escritor para lo cual se le atribuiría un valor entre 1 y 6 en función de una serie de características descritas en la Tabla 1.

TABLA 1.

Medidas para evaluar la planificación textual (Limpo y Alves, 2013).

\begin{tabular}{|c|c|c|}
\hline Tipo de planificación & Características & Puntuación \\
\hline $\begin{array}{l}\text { Ausencia de } \\
\text { planificación }\end{array}$ & $\begin{array}{l}\text { La planificación es exactamente igual al texto o existen diferencias } \\
\text { mínimas. } \\
\text { Apenas una o dos palabras están escritas. } \\
\text { La hoja está en blanco. } \\
\text { La planificación no está relacionada con la instrucción. } \\
\text { La planificación es un conjunto de temas que se copian en el texto sin } \\
\text { ser desarrollado. } \\
\text { De la lista de frases o palabras menos de la mitad corresponde al texto. }\end{array}$ & 1 \\
\hline Planificación mínima & $\begin{array}{l}\text { La planificación coincide con el texto, pero hay cambios menores en el } \\
\text { contenido, la estructura y el orden de las frases (comparando texto con } \\
\text { borrador son similares, pero el estudiante demuestra que no sólo copia, } \\
\text { sino que para el texto trató de mejorar algo lo del borrador). }\end{array}$ & 2 \\
\hline Resumen & La planificación es un resumen del texto. & 3 \\
\hline Lista & $\begin{array}{l}\text { Lista de preguntas, frases, palabras o temas que se desarrollan } \\
\text { mínimamente en el texto, pero hay pocos detalles (no hay otros } \\
\text { subtemas). } \\
\text { Los temas pueden tener contenidos (introducción: coherente) o no (por } \\
\text { ejemplo: una opinión ). }\end{array}$ & 4 \\
\hline $\begin{array}{l}\text { Subordinación } \\
\text { emergente }\end{array}$ & $\begin{array}{l}\text { Temas con poco o ningún detalle relativo sobre las partes del texto, } \\
\text { pero organizado de acuerdo a una estructura explícita (la introducción / } \\
\text { primera y segunda parte del desarrollo / conclusión) Puede tener algo o } \\
\text { poco de contenido. }\end{array}$ & 5 \\
\hline $\begin{array}{l}\text { Relaciones } \\
\text { estructurales }\end{array}$ & $\begin{array}{l}\text { Los temas están muy bien desarrollados y la estructura es explícita } \\
\text { (esquema, organización) tiene las partes desarrollados. }\end{array}$ & 6 \\
\hline
\end{tabular}

Fuente: Autores.

Las medidas del producto textual se obtuvieron al evaluar las hojas del texto final. Para ello se utilizaron las Medidas Basadas en el Texto (MBT) y Medidas Basadas en el Lector (MBL) descritas en la Tabla 2. 
TABLA 2.

Medidas Basadas en el Texto y Medidas Basadas en el Lector usadas para evaluar el producto textual.

\begin{tabular}{|c|c|c|c|}
\hline & Ítem & Características & Puntuación \\
\hline & $\begin{array}{l}\text { Productividad } \\
\text { Organización } \\
\text { de la } \\
\text { información }\end{array}$ & $\begin{array}{l}\text { Número de párrafos. } \\
\text { Número de palabras. } \\
\text { Coherencia referencial: indicadores referenciales y lexicales } \\
\text { Coherencia relacional: meta-estructurales, estructuradores, } \\
\text { conectores, reformuladores y argumentativos. }\end{array}$ & $\begin{array}{l}\text { Contabilizar } \\
\text { cada elemento }\end{array}$ \\
\hline $\begin{array}{l}\text { Medidas } \\
\text { Basadas } \\
\text { en el } \\
\text { Texto }\end{array}$ & Estructura & $\begin{array}{l}\text { Para textos de comparación y contraste: } \\
\text { - Si hay una introducción que presente el tema o el punto de vista } \\
\text { del autor se dará } 1 \text { punto, si no la hay } 0 \text { puntos. } \\
\text { - Si hay un cuerpo de explicación y desarrollo en el texto se } \\
\text { asignará } 1 \text { punto, en caso contrario } 0 \text { puntos. } \\
\text { - Si hay una conclusión que de una visión del conjunto } 1 \text { punto, si } \\
\text { no existe } 0 \text { puntos. } \\
\text { Para textos narrativos (se suma un punto por cada elemento } \\
\text { presente): tiempo, espacio, personajes, suceso inicial, respuesta } \\
\text { interna, ejecución y consecuencias. }\end{array}$ & $\begin{array}{l}\text { 1: No } \\
\text { estructurada } \\
\text { 2: Pobremente } \\
\text { estructurada } \\
\text { 3: Parcialmente } \\
\text { estructurada } \\
\text { 4: Bien } \\
\text { estructurada }\end{array}$ \\
\hline \multirow{3}{*}{$\begin{array}{l}\text { Medidas } \\
\text { Basada } \\
\text { en el } \\
\text { Lector }\end{array}$} & Estructura & $\begin{array}{l}\text { Para los textos de comparación y contraste: } \\
\text { - Información sobre un marco o introducción que presenta el texto. } \\
\text { - Marcas o señales de estructura. } \\
\text { - Una introducción que presenta el objetivo y tema del texto, la } \\
\text { comparación y contraste entre los temas. } \\
\text { - Un desarrollo organizado y estructurado, por ejemplo: agrupando } \\
\text { en un bloque las semejanzas y en otro bloque las diferencias. } \\
\text { - Una unidad entre los párrafos. } \\
\text { - Una conclusión final al texto. } \\
\text { Para los textos narrativos: } \\
\text { - Información del marco que desarrolle el contexto. } \\
\text { - Marcas o señales de estructura. } \\
\text { - Un suceso que cree un propósito, problema o complicación para el } \\
\text { principal protagonista. } \\
\text { - Esfuerzo del protagonista para resolver el suceso. } \\
\text { - Resultados de los esfuerzos del protagonista } \\
\text { Una relación causal o temporal de los eventos. }\end{array}$ & $\begin{array}{l}\text { 1: No } \\
\text { estructurada } \\
\text { 2: Pobremente } \\
\text { estructurada } \\
\text { 3: Parcialmente } \\
\text { estructurada } \\
\text { 4:Bien } \\
\text { estructurada }\end{array}$ \\
\hline & Coherencia & $\begin{array}{l}\text { Identificación del tema. } \\
\text { La exposición del tema sin digresiones } \\
\text { Un contexto el cual oriente al lector. } \\
\text { Los detalles se organizan en un plan distinguible el cual es } \\
\text { sustentado a lo largo de todo el texto. } \\
\text { Existen marcas o enlaces que cohesionan oraciones y párrafos. } \\
\text { El discurso fluye sin problemas. } \\
\text { La existencia de una conclusión que dé sentido de final al texto. }\end{array}$ & $\begin{array}{l}\text { 1: Incoherente } \\
\text { 2: Próximo a la } \\
\text { incoherencia } \\
\text { 3: Algo } \\
\text { coherente } \\
\text { 4: Muy } \\
\text { coherente }\end{array}$ \\
\hline & Calidad & $\begin{array}{l}\text { Una clara secuencia de ideas. } \\
\text { Buena organización } \\
\text { Vocabulario adecuado. } \\
\text { Variedad de detalles } \\
\text { Correcta estructura de las oraciones } \\
\text { Correcta puntuación y ortografía. }\end{array}$ & $\begin{array}{l}\text { 1: Inadecuado, } \\
\text { difícil } \\
\text { comprensión } \\
\text { 2: Apenas } \\
\text { adecuado } \\
\text { 3: Adecuado } \\
\text { 4: Bueno } \\
\text { 5: Muy bueno } \\
\text { 6: Excelente }\end{array}$ \\
\hline
\end{tabular}

Fuente: Autores. 
Para evaluar el proceso de revisión textual, una vez elaborados los textos se valoraron los dos subprocesos generales que lo componen, la detección y la corrección de errores, siguiendo para ello dos fases de análisis. En un primer momento, se entregaron a los alumnos los textos que habían escrito, y se les pidió que los leyeran para detectar los errores cometidos. Se les entregó una hoja en la que debían explicar los errores encontrados y dar una solución a los mismos, conociendo así las estrategias que los niños utilizaban a la hora de corregir un error. Durante esta primera fase, se activaba el proceso de detección de errores, que se evaluó haciendo un recuento de los errores, tanto mecánicos como sustantivos, que había cometido el alumno y el número de errores que este detectaba correctamente, hallándose posteriormente este porcentaje.

Para la segunda fase, se entregó a cada niño su propio texto en el que los errores ya estaban señalados, y otra hoja en la que debían escribir la solución a dichos errores, evaluando así el proceso de corrección. Para esta evaluación, se realizó una categorización de las estrategias de corrección empleadas en base a un sistema de elaboración propio que consistía en contabilizar el número de veces en las cuales los alumnos empleaban cada estrategia, concretamente para valorar las siguientes estrategias: añadir, quitar, reordenar o cambiar, para solucionar los errores marcados y se calculó el porcentaje de las veces que esta fue empleada correctamente para solucionar tanto errores mecánicos como sustantivos.

\section{Diseño de la investigación}

El trabajo de campo consistió en 4 sesiones de evaluación cada una de ellas de una duración aproximada de una hora, que se llevaron a cabo en la propia aula escolar y fueron desarrolladas por parte de personal científico cualificado, aunque siempre con la presencia de un maestro del centro, que no intervino en ningún momento de la investigación. En la primera sesión, los alumnos elaboraron un texto narrativo de temática libre y en la segunda un texto de comparación y contraste cuya temática se asignó de manera aleatoria para evitar posibles efectos sobre los resultados del tema sobre el que escribir; las temáticas textuales en este caso fueron: "Diferencias y semejanzas entre el fútbol y el baloncesto" y "Diferencias y semejanzas entre las vacaciones de verano y vacaciones de Navidad". Para ambas sesiones se les facilitó a los alumnos dos hojas, una de ellas como borrador para, de manera opcional, hacer una planificación, y otra para escribir el texto final.

Las otras dos sesiones de la investigación se dedicaron a la evaluación de los procesos de revisión textual. De forma que la tercera sesión se destinó a evaluar los procesos de revisión para el texto narrativo y la cuarta sesión para el texto de comparación y contraste. Cada una de las dos sesiones se organizó en dos tiempos: Para la primera parte de la sesión se dedicaron aproximadamente 15 minutos y se centró en la fase de detección de errores; para ello se entregó a los alumnos el texto que ellos mismos habían elaborado y se les pidió que realizaran una lectura del mismo en busca de errores que, una vez encontrados, debían marcar, explicar y solucionar. La segunda parte de la sesión se dedicó a la fase de corrección de errores y se emplearon los 45 minutos restantes de la sesión; para evaluar esta fase se entregaron a los alumnos sus textos con los errores ya señalados y ellos únicamente debían corregirlos en otra hoja. Los errores se marcaron siguiendo un código de colores y letras que fueron explicados a los alumnos con una leyenda. Así, estarían rodeados en rojo los errores 
referentes a aspectos mecánicos como: la Ortografía $(\mathrm{O})$, abarcando faltas de ortografía o acentuación; los errores de Gramática $(\mathrm{G})$, como errores de conjugación, género y número; y los errores de Puntuación (P) cuando el uso de los signos de puntuación era erróneo. Por otro lado, la ausencia de algún elemento necesario para la comprensión del texto fue señalada con un asterisco de color azul, siendo estos elementos signos de puntuación (P), palabras (Palabra), ideas (Idea) o frases (Frase). Finalmente, los errores sustantivos se subrayaron en amarillo, considerándolos como errores cuando un elemento sobra en el texto (Innecesario), no tiene sentido o le falta lógica (Sin sentido), es de difícil comprensión (Lioso), o cuando el orden de las frases o sucesos es inadecuado (Desordenado).

Una vez finalizado el trabajo de campo, un corrector experto entrenado previamente en los sistemas de corrección, corrigió las tareas realizadas por los alumnos. Los datos obtenidos fueron informatizados y analizados estadísticamente, obteniéndose los resultados que se analizan a continuación.

\section{ANÁLISIS DE RESULTADOS}

Para analizar los resultados que se presentan a continuación se utilizó el programa estadístico de datos Statistical Package for the Social Sciences (SPSS, versión 24.0).

Para comprobar si la muestra cumplía los criterios de normalidad en cada variable objeto de estudio, se realizaron pruebas descriptivas de asimetría y curtosis, pudiendo determinar si convenía realizar análisis paramétrico o no paramétrico. Los datos obtenidos se recogen en la Tabla 3, y en ella se muestran qué medidas de productividad, producto, planificación y revisión cumplen los criterios de normalidad y cuáles no. Se han considerado como criterios aceptables de asimetría y curtosis valores de asimetría entre \pm 2 y de curtosis entre \pm 7 (Finney \& Distefano, 2006).

TABLA 3.

Normalidad de la muestra según asimetría y curtosis para medidas de producto textual, planificación y detección de errores.

\begin{tabular}{llllc}
\hline \multirow{2}{*}{ Variables Asimetría } & \multicolumn{2}{c}{ Texto de comparación y contraste } & \multicolumn{2}{c}{ Texto narrativo } \\
\cline { 2 - 5 } & \multicolumn{2}{c}{ Curtosis } & Asimetría & Curtosis \\
\hline $\mathrm{N}^{\mathrm{o}}$ párrafos & 13.813 & 213.052 & 2.789 & 16.728 \\
Coherencia referencial & 1.107 & 2.641 & 1.040 & 1.838 \\
Coherencia relacional & 1.041 & 1.774 & .757 & .704 \\
Estructura MBT & .927 & -0.144 & -0.453 & -0.492 \\
Estructura MBL & 1.346 & 2.609 & -0.070 & -0.731 \\
Coherencia MBL & 1.118 & .898 & -0.184 & -0.725 \\
Calidad MBL & .975 & .521 & -0.208 & -0.978 \\
MBL total & 1.367 & 1.756 & -0.189 & -0.868 \\
No palabras del borrador & .602 & -0.755 & 4.103 & 41.637 \\
Tipo de planificación & 3.567 & 14.425 & 3.175 & 15.101 \\
Errores mecánicos & 1.220 & 1.971 & 1.686 & 5.129 \\
Errores mecánicos detectados & 1.450 & 2.118 & 1.889 & 5.632
\end{tabular}




\begin{tabular}{|c|c|c|c|c|c|}
\hline \multirow{8}{*}{$\begin{array}{l}\text { Estrategias } \\
\text { corrección } \\
\text { errores } \\
\text { mecánicos }\end{array}$} & Estrategia añadir & -0.122 & -0.1024 & -0.153 & -.660 \\
\hline & Estrategia añadir utilidad & 0.035 & -1.056 & 0.095 & -.657 \\
\hline & Estrategia quitar & 2.132 & 6.151 & 2.550 & 9.054 \\
\hline & Estrategia quitar utilidad & 2.451 & 7.013 & 2.245 & 7.114 \\
\hline & Estrategia reordenar & 0.361 & -0.242 & 2.958 & 10.493 \\
\hline & Estrategia reordenar utilidad & 0.410 & -0.424 & 2.659 & 8.913 \\
\hline & Estrategia cambiar & 0.973 & 0.514 & 1.338 & 1.600 \\
\hline & Estrategia cambiar utilidad & 1.044 & 0.834 & 1.654 & 3.626 \\
\hline \multicolumn{2}{|c|}{ Errores sustantivos } & 4.024 & 17.885 & 10.008 & 125.694 \\
\hline \multicolumn{2}{|c|}{ Errores sustantivos detectados } & 2.388 & 4.011 & 1.982 & 2.220 \\
\hline \multirow{8}{*}{$\begin{array}{l}\text { Estrategias } \\
\text { corrección } \\
\text { errores } \\
\text { sustantivos }\end{array}$} & Estrategia añadir & 0.881 & -1.254 & -1.688 & 1.582 \\
\hline & Estrategia añadir utilidad & 0.881 & -1.254 & -0.056 & -1.815 \\
\hline & Estrategia quitar & -1.252 & -0.137 & -1.590 & 1.472 \\
\hline & Estrategia quitar utilidad & -0.922 & -0.947 & -1.064 & -0.367 \\
\hline & Estrategia reordenar & -1.113 & -0.652 & -1.827 & 2.110 \\
\hline & Estrategia reordenar utilidad & -0.984 & -0.991 & -1.652 & 1.355 \\
\hline & Estrategia cambiar & -0.565 & -1.335 & 1.293 & . \\
\hline & Estrategia cambiar utilidad & -0.768 & -0.818 & 1.293 & . \\
\hline
\end{tabular}

Fuente: Autores.

Después de hallar la asimetría y la curtosis de las variables se decidió realizar dos tipos de análisis: paramétricos para aquellas variables que cumplen los criterios de normalidad, y análisis no paramétricos para aquellas que no cumplen los supuestos de normalidad. Con la intención de cumplir con los objetivos y comprobar las hipótesis planteadas inicialmente en el estudio, se presentan a continuación los análisis estadísticos realizados y los resultados obtenidos.

Para afrontar el objetivo de este articulo se consideraron como variable independiente el curso al que pertenecen los alumnos $\left(4^{\circ}, 5^{\circ}\right.$ y $6^{\circ}$ de EPO) y como variables dependientes las diferentes medidas de producto, planificación y detección de errores y se realizaron, por un lado, pruebas paramétricas de análisis de varianza (ANOVA y MANOVA). Por otro lado pruebas no paramétricas, como la Prueba H de Kruskal-Wallis y la Prueba U de Mann-Whitney, tomando como variable de agrupamiento el curso de los alumnos y relacionándola con las diferentes medidas de producto, planificación y detección de errores.

\section{Análisis comparativo de la calidad textual y las habilidades de planificación y revisión en función del curso de los alumnos.}

Centrándonos en los análisis del producto textual, se realizó un análisis paramétrico multivariado MANOVA, el cual mostró la existencia de diferencias estadísticamente significativas $\left[\mathrm{F}_{(24,488)}=6.078, \mathrm{p}<0.001, \mathrm{n}^{2}=0.230\right]$. Con las pruebas de efectos inter-sujetos se observó que los cursos diferían en las MBL en cuanto a coherencia y calidad y el total de las medidas de MBL para los textos de comparación y contraste, y en las MBL de estructura, coherencia y calidad y el total de MBL para los textos narrativos (Tabla 4). 
TABLA 4.

Resultados estadísticamente significativos del MANOVA, para medidas de producto textual.

\begin{tabular}{|c|c|c|c|c|c|c|c|c|c|c|}
\hline \multirow{2}{*}{\multicolumn{2}{|c|}{ Variables M }} & \multicolumn{2}{|c|}{$4^{\circ}$} & \multicolumn{2}{|c|}{$5^{\circ}$} & \multicolumn{2}{|c|}{$6^{\circ}$} & \multirow{2}{*}{$\mathrm{F}$} & \multirow{2}{*}{$\mathrm{p}$} & \multirow{2}{*}{$\eta 2$} \\
\hline & & DT & M & DT & M & $\mathrm{DT}$ & & & & \\
\hline \multirow{3}{*}{$\begin{array}{l}\text { Comparación } \\
\text { y contraste }\end{array}$} & Coherencia MBL & 1.30 & .488 & 1.56 & 0.714 & 1.84 & .786 & 13.007 & 0.000 & 0.093 \\
\hline & Calidad MBL & 1.60 & 0.748 & 2.20 & 1.239 & 2.59 & 1.121 & 17.798 & 0.000 & 0.122 \\
\hline & Total MBL & 4.04 & 1.371 & 5.10 & 2.373 & 5.70 & 2.315 & 13.078 & 0.000 & 0.093 \\
\hline \multirow{5}{*}{$\begin{array}{l}\text { Textos } \\
\text { narrativos }\end{array}$} & Estructura MBL & 2.26 & 0.785 & 2.80 & 0.815 & 3.10 & 0.774 & 23.585 & 0.000 & 0.156 \\
\hline & Coherencia MBL & 2.31 & 0.847 & 2.82 & 0.779 & 3.24 & 0.711 & 29.144 & 0.000 & 0.186 \\
\hline & Calidad MBL & 3.25 & 1.397 & 3.90 & 1.234 & 4.50 & 1.194 & 19.942 & 0.000 & 0.135 \\
\hline & Total MBL & 7.82 & 1.708 & 9.52 & 2.632 & 10.84 & 2.495 & 27.583 & 0.000 & 0.178 \\
\hline & Número de palabras & 134.12 & 50.941 & 127.80 & 40.450 & 151.40 & 45.099 & 6.908 & 0.001 & 0.048 \\
\hline
\end{tabular}

Fuente: Autores.

Para las variables en las que se habían encontrado diferencias estadísticamente significativas, se realizó contraste post hoc, con el fin de comprobar entre qué grupos se producían estas diferencias, observando los resultados que se recogen en la Tabla 5.

TABLA 5.

Contrastes post hoc en las medidas de producto textual.

\begin{tabular}{|c|c|c|c|c|}
\hline \multicolumn{2}{|c|}{ Variables } & \multicolumn{2}{|l|}{ Curso } & \multirow{3}{*}{$\frac{\mathrm{p}}{0.048}$} \\
\hline \multirow{6}{*}{$\begin{array}{l}\text { Comparación } \\
\text { y contraste }\end{array}$} & \multirow{2}{*}{ Coherencia MBL } & 4 & 5 & \\
\hline & & 6 & 0.000 & \\
\hline & \multirow{2}{*}{ Calidad MBL } & 4 & 5 & \multirow[t]{2}{*}{0.001} \\
\hline & & 6 & 0.000 & \\
\hline & \multirow{2}{*}{ Total MBL } & 4 & 5 & \multirow[t]{2}{*}{0.005} \\
\hline & & 6 & 0.000 & \\
\hline \multirow{15}{*}{$\begin{array}{l}\text { Textos } \\
\text { narrativos }\end{array}$} & \multirow{3}{*}{$\begin{array}{l}\text { Estructura MBL } \\
5\end{array}$} & 4 & 5 & \multirow[t]{3}{*}{0.000} \\
\hline & & 6 & 0.000 & \\
\hline & & 6 & 0.035 & \\
\hline & \multirow{3}{*}{$\begin{array}{l}\text { Coherencia MBL } \\
5\end{array}$} & 4 & 5 & \multirow[t]{3}{*}{0.000} \\
\hline & & 6 & 0.006 & \\
\hline & & 6 & 0.002 & \\
\hline & \multirow{3}{*}{$\begin{array}{l}\text { Calidad MBL } \\
5\end{array}$} & 4 & 5 & \multirow[t]{3}{*}{0.004} \\
\hline & & 6 & 0.000 & \\
\hline & & 6 & 0.008 & \\
\hline & \multirow{3}{*}{$\begin{array}{l}\text { Total MBL } \\
5\end{array}$} & 4 & 5 & \multirow[t]{3}{*}{0.000} \\
\hline & & 6 & 0.000 & \\
\hline & & 6 & 0.003 & \\
\hline & \multirow{3}{*}{$\begin{array}{l}\text { Número de palabras } \\
5\end{array}$} & 4 & 5 & \multirow[t]{3}{*}{ s.n. } \\
\hline & & 6 & 0.010 & \\
\hline & & 6 & 0.002 & \\
\hline
\end{tabular}

Fuente: Autores. 
Tal y como se observa en la Tabla 5, para todas las variables había diferencias significativas entre los alumnos de $4^{\circ}$ y los de $5^{\circ}$ y $6^{\circ}$. De esta forma se comprobó que, en todos los casos, los alumnos de $4^{\circ}$ puntuaban con medias inferiores a las de los alumnos de cursos superiores. Sin embargo, se puede observar que no hay diferencias significativas al comparar a los alumnos de $5^{\circ}$ con los de $6^{\circ}$ en los textos de comparación y contraste, aunque si las haya en los textos narrativos, donde son los alumnos de $6^{\circ}$ los que obtienen medias con mejor puntuación en todas las variables de MBL.

Para finalizar con el análisis del producto textual, para las variables que no se ajustaban a los criterios de normalidad se realizó la Prueba $\mathrm{H}$ de Kruskal-Wallis, la cual indicó que existen diferencias significativas entre los cursos en cuanto al número de palabras escritas en el texto de comparación y contraste $\left(\mathrm{x}^{2}=15.458, \mathrm{p}<0.001\right)$ y en el número de párrafos en el texto narrativo $\left(x^{2}=56.910, p<0.001\right)$. Se realizó la Prueba U de Mann-Whitney para comparar los cursos como grupos dos a dos, y se encontraron diferencias significativas en la variable número de párrafos redactados en el texto narrativo entre los alumnos de $4^{\circ} \mathrm{y}$ $5^{\mathrm{o}}(\mathrm{Z}=2088.0, \mathrm{p}<0.001)$ y entre los de $4^{\circ}$ y $6^{\circ}(\mathrm{Z}=1693.00, \mathrm{p}<0.001)$, observándose en ambos casos que los alumnos de $4^{\circ}$ los que organizaban la información del texto en un menor número de párrafos $\left(\mathrm{RP} 4^{\circ}=87.48, \mathrm{RP}^{\circ}=155.07, \mathrm{RP}^{\circ}=169.98\right)$.

También se encontraron diferencias significativas entre los alumnos de $4^{\circ}$ y $5^{\circ}$ en cuanto a la variable de número de palabras escritas en el texto de comparación y contraste entre $4^{\circ}$ y $5^{\circ}(Z=3119.5, p=0.022)$ y entre $4^{\circ}$ y $6^{\circ}(Z=2543.50, p<0.001)$, siendo una vez más en ambos casos los alumnos de $4^{\circ}$ eran los que menor rango promedio poseían $\left(\mathrm{RP} 4^{\circ}=110.23\right.$, $\left.R P 5^{\circ}=135.82, \mathrm{RP}^{\circ}=156.51\right)$, lo cual significa que estos alumnos producían menos que los alumnos de cursos superiores.

Una vez analizado el producto textual se procedió a realizar evaluar los procesos textuales de planificación y revisión textual empleados por los alumnos.

Centrándonos en primer lugar en el procesos de planificación, se utilizó un análisis ANOVA para la variable de número de palabras escritas en el borrador empleado para el texto de comparación y contraste, encontrándose diferencias estadísticamente significativas entre los cursos educativos $\left(\mathrm{F}=5.547, \mathrm{p}=004, \mathrm{n}^{2}=0.040\right)$, se procedió, por lo tanto, a realizar contrastes post hoc para concretar entre qué grupos se producían estas discrepancias, obteniéndose diferencias estadísticamente significativas entre $5^{\circ}$ y $6^{\circ}\left(\mathrm{M}^{\circ}=35.38\right.$ vs. $\mathrm{M}^{\circ}=59,21, \mathrm{p}=$ 0.005 ), de modo que los alumnos de $6^{\circ}$ son los que generaban un mayor número de ideas en el borrador del texto de comparación y contraste, y los alumnos de $5^{\mathrm{o}}$ los que menos.

Para aquellas variables del proceso de planificación textual que no se ajustaban a los criterios de normalidad, se empleó la Prueba H de Kruskal-Wallis, cuyo análisis muestra la existencia de diferencias estadísticamente significativas entre los cursos en las variables de tipo de planificación y número de palabras escritas en el borrador del texto narrativo $\left(\mathrm{X}^{2}=6.269, \mathrm{p}=0.044 ; \mathrm{X}^{2}=14.154, \mathrm{p}=0.001\right.$ respectivamente). Dichas diferencias se concretaron con la Prueba U de Mann, mostrando que existen diferencias estadísticamente significativas en la variable del tipo de planificación realizada para los textos narrativos entre los alumnos de $4^{\circ}$ y $6^{\circ}(Z=3532.00, p=0.021)$ y entre $5^{\circ}$ y $6^{\circ}(Z=4109.50, p=0.025)$, siendo en ambos casos los alumnos de $6^{\circ}$ los que menos planificaban sus textos, puesto que su rango promedio era menor que el de los alumnos de $4^{\circ}$ y $5^{\circ}\left(\mathrm{RP} 4^{\circ}=145.79, \mathrm{RP}^{\circ}=144.77\right.$, $\left.\mathrm{RP}^{\circ}=128.44\right)$. También se encontraron diferencias significativas en la variable de número 
de ideas generadas en el borrador del texto narrativo entre $4^{\circ}$ y $5^{\circ}(Z=2958.00, p=001)$ y entre $5^{\circ}$ y $6^{\circ}(Z=3480.50, p=0.002)$, siendo en ambos casos los alumnos de $5^{\circ}$ curso los que menor rango promedio tenían $\left(\mathrm{RP} 4^{\circ}=155.63, \mathrm{RP}^{\circ}=115.63, \mathrm{RP} 6^{\circ}=150.14\right)$, lo cual significa que los alumnos de este curso generaban menos ideas en el borrador del texto narrativo que los otros dos cursos comparados.

Finalmente, en lo referente al subproceso de detección de errores propio del proceso de revisión, se llevó a cabo un análisis univariado de varianza (ANOVA), que permitió comprar la existencia de diferencias estadísticamente significativas en cuanto a la variable de detección de errores mecánicos en los textos de comparación y contraste $(\mathrm{F}=3.866, \mathrm{p}=0.022$, $\left.\eta^{2}=0.029\right)$ cuyas pruebas post hoc muestran que estas diferencias son significativas entre $4^{\mathrm{o}}$ y $5^{\mathrm{o}}\left(\mathrm{M} 4^{\mathrm{o}}=22.21 \mathrm{vs} . \mathrm{M} 5^{\mathrm{o}}=14.082, \mathrm{p}=0.044\right)$, siendo los alumnos de $4^{\circ}$ los que mayor número de errores mecánicos detectaban en este tipo de textos. Tras realizar análisis no paramétricos de las variables que no se ajustaban a los parámetros de normalidad, no se obtuvieron diferencias significativas entre los cursos en cuanto a las habilidades de revisión textual en los textos de comparación y contraste para las variables de errores sustantivos y detección de los mismos, así como para la variable de errores sustantivos de los textos narrativos, lo cual quiere decir que los alumnos de $4^{\circ}, 5^{\circ}$ y $6^{\circ}$ poseen habilidades similares en cuanto a su capacidad para detectar errores de tipo sustantivo.

\section{Análisis descriptivo de las estrategias de detección y corrección de errores durante el proceso de revisión}

Para comprobar qué estrategias de corrección de errores utilizan los alumnos con mayor frecuencia y si estas varían a medida que crecen los alumnos, se realizaron análisis descriptivos. Estos se presentan de manera comparativa en función del curso a través de las medias de los porcentajes de veces que los alumnos empleaban cada estrategia para la corrección de errores mecánicos y sustantivos en textos de comparación contraste (Figura 1) y narrativos (Figura 2).

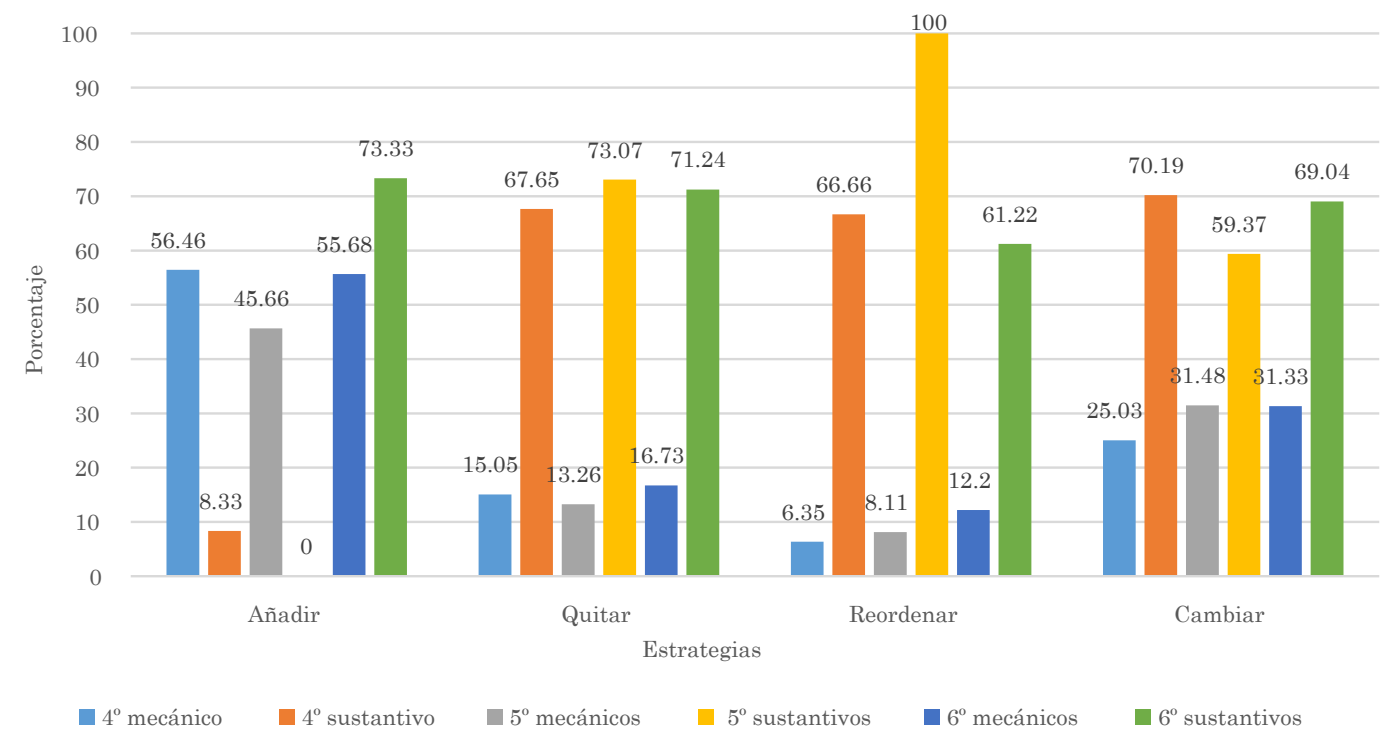

Figura 1. Estrategias de corrección de errores en textos de comparación y contraste. Fuente: Autores. 
Tal y como se puede observar en la Figura 1, en caso de textos de comparación-contraste los alumnos de $4^{\circ}$ curso empleaban con mayor frecuencia la estrategia de añadir para corregir errores mecánicos y con menor frecuencia la estrategia de reordenar. Por su parte, ante la corrección de errores sustantivos, estos alumnos empleaban más veces la estrategia de cambiar seguida de cerca por las estrategias de quitar y reordenar siendo, por lo tanto, la estrategia de añadir la menos utilizada. En los textos narrativos (Figura 2) sucedió lo mismo en cuanto a los errores mecánicos y lo contrario en relación a los sustantivos ya que los alumnos usaban en mayor medida la estrategia de añadir y en menor medida cambiar.

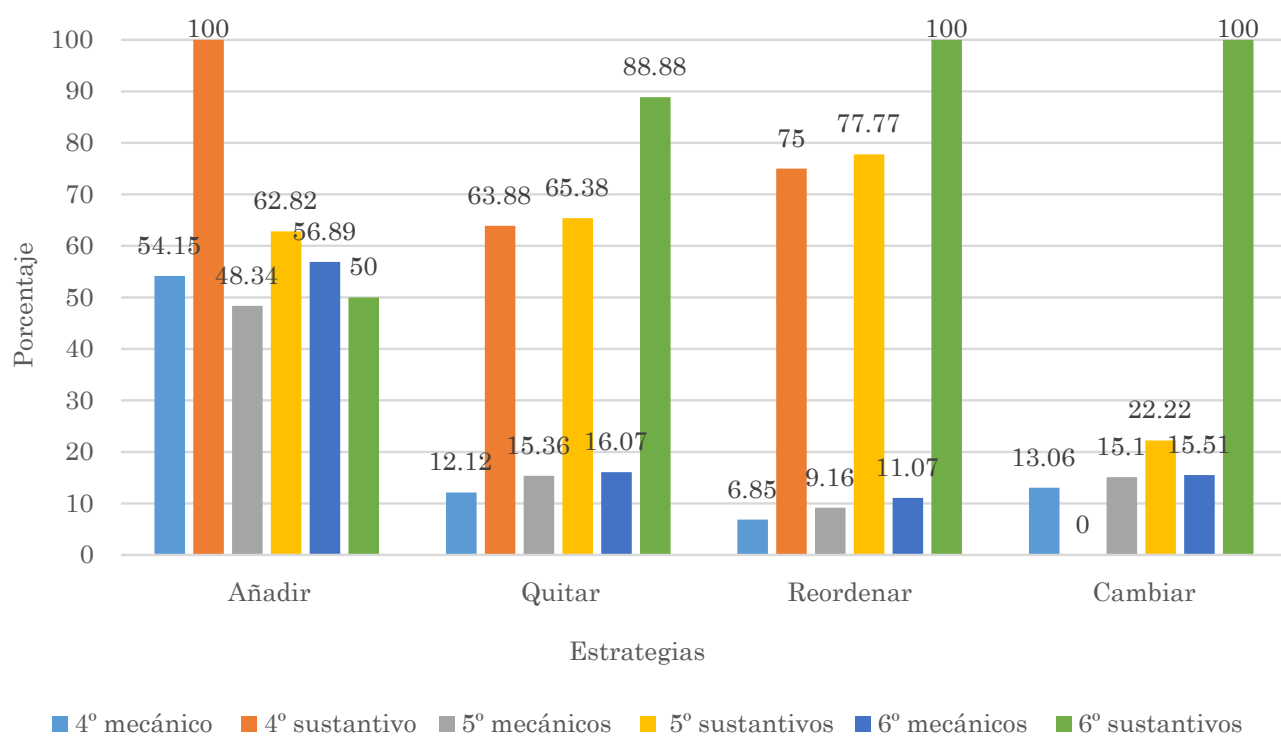

Figura 2. Estrategias de corrección de errores en textos narrativos. Fuente: Autores.

Por su parte, los alumnos de $5^{\circ}$ recurrían más frecuentemente a la estrategia de añadir y en menor medida la estrategia de reordenar para la corrección de errores mecánicos. Mientras que los errores sustantivos eran corregidos en más ocasiones con la estrategia de reordenar, siendo las estrategias menos utilizadas las de añadir, en el caso de los textos de comparación y contraste, y cambiar en el caso de los textos narrativos.

$\mathrm{Al}$ igual que los alumnos de $4^{\circ}$ y $5^{\circ}$, cuando los alumnos de $6^{\circ}$ debían corregir un error mecánico, empleaban la estrategia añadir, frente a la estrategia de reordenar la que menos se utilizó. Sin embargo en cuanto a las estrategias utilizadas para la corrección de errores sustantivos se observan diferencias en ambos tipos de textos examinados, empleándose en mayor medida la estrategia de quitar (aunque la estrategia de añadir se utilizó casi con el mismo porcentaje de uso), y en menor medida, la estrategia de reordenar en los textos de comparación y contraste; y utilizándose un mayor número de veces las estrategias de reordenar y cambiar, en los textos narrativos, siendo la estrategia de añadir la menos utilizada.

Recapitulando, la corrección de errores mecánicos los alumnos de todos los cursos empleaban un número más elevado de veces la estrategia de añadir, mientras que la estrategia reordenar fue la menos utilizada. Sin embargo, se observan diferencias a la hora de corregir los errores sustantivos en función del texto. Así, en los textos de comparación y contraste para los alumnos de $4^{\circ}$ la estrategia más utilizada era cambiar, para los de $5^{\circ}$ era reordenar y para los de $6^{\circ}$ era quitar. En cuanto a las estrategias que obtuvieron un menor porcentaje de uso, los alumnos de $4^{\mathrm{o}}$ y $5^{\mathrm{o}}$ coinciden en utilizar un menor número de veces la estrategia de añadir para corregir 
este tipo de errores, mientras que los de $6^{\circ}$ utilizan en menor medida la estrategia de reordenar. Por su parte, la corrección de errores sustantivos en los textos narrativos, los alumnos de $4^{\circ}$ se decantaban por añadir y los de $5^{\circ}$ por reordenar, frente a cambiar, que fue la estrategia menos utilizada en ambos cursos. Los alumnos de $6^{\circ}$ utilizaban el mismo número de veces las estrategias de añadir, reordenar y cambiar, pero la estrategia añadir fue la que menos errores solucionó tras ser activada.

\section{CONCLUSIONES}

El objetivo del estudio era analizar comparativamente las habilidades y estrategias de escritura o composición escrita relativas al proceso de planificación textual y al de revisión textual que presentan el alumnado español de $4^{\circ}, 5^{\circ}$ y $6^{\circ}$ curso de Primaria. Una de las hipótesis planteadas fue que existirían estas diferencias, prediciendo que los alumnos de $6^{\circ}$ serían quienes lograrán textos de mayor calidad, puesto que se presuponía que serían los que más planificarían y revisarían sus textos. Esta hipótesis se cumple en parte, puesto que si bien es cierto que los alumnos de $6^{\circ}$ eran los que mejores productos textuales elaboraron, no siempre fueron los que más habilidades de planificación y revisión desplegaron.

Atendiendo al producto textual, se observó que los textos que elaboraban los alumnos de $4^{\circ}$ curso de EPO puntuaban más bajo en cuanto a las medidas de estructura, coherencia y de calidad en textos narrativos y las mismas medidas salvo estructura para los textos de comparación y contraste. También se aprecia que estos alumnos son los que menos palabras escriben en los textos de comparación y contraste y los que estructuran el texto en menos párrafos que los alumnos de cursos superiores. De modo que se puede concluir que los alumnos de $4^{0}$ aún no han desarrollado del todo las habilidades necesarias para elaborar textos coherentes y de calidad. Por su parte, los alumnos de $6^{\circ}$ fueron los que elaboraron textos con mayor puntuación en las medidas de estructura, coherencia y calidad basadas en la percepción subjetiva del lector.

En lo referente a los procesos de planificación, se observa que el tipo de planificación que la generalidad de los alumnos estudiados realizan es una planificación mínima, basada fundamentalmente en escribir un texto en el borrador y copiarlo literalmente como composición textual final; si bien se observa que el alumnado mayor es el que más productividad evidencia en sus borradores, siendo por lo tanto los alumnos de $5^{\circ}$ y $6^{\circ}$ curso los que más ideas previas al texto final generan.

Por su parte, tras comprobar los resultados de los procesos de revisión, concretamente el subproceso de detección de errores, se puede concluir que los alumnos de $4^{\circ}$ cometían un mayor número de errores mecánicos en los textos narrativos y que, a su vez, eran los que más errores de este tipo detectaban en los textos de comparación y contraste. Por otro lado, no se encontraron diferencias estadísticamente significativas entre los cursos en cuanto a las habilidades de revisión desplegadas para la detección de errores sustantivos del texto.

En cuanto a las estrategias de corrección de errores que los alumnos de los diferentes cursos activaban ante una tarea de revisión real se confirma que los alumnos de todos los cursos tienen una clara preferencia por la estrategia de añadir contenido, mientras que la estrategia de reordenar fue la menos usada para corregir errores mecánicos. No obstante, se observan discrepancias en lo referente a los errores sustantivos, donde las estrategias empleadas varían entre los diferentes cursos. Así, los alumnos de $4^{\circ}$ emplearían un mayor número de veces la estrategia cambiar frente a añadir para corregir el error en el caso de los textos de comparación 
y contraste, aunque estas preferencias de uso se invierten en los textos narrativos. Por su parte, los alumnos de $5^{\circ}$ recurren más a la estrategia de reordenar en ambas tipologías textuales, mientras que añadir y cambiar fueron las menos usadas para los textos de comparación y contraste y textos narrativos respectivamente. En los alumnos de $6^{0}$ se observa una mayor variación de estrategias en función del tipo de texto. Así, los errores sustantivos presentes en los textos de comparación y contraste son corregidos quitando el contenido erróneo, y siendo reordenar la estrategia menos empleada, mientras que en los textos narrativos, los alumnos de este curso preferían reordenar y cambiar antes que añadir el contenido.

Así pues, tras analizar el producto escrito y las habilidades de planificación y revisión textual que presenta el alumnado hispano de diferentes cursos, se concluye que la adquisición de las habilidades cognitivas superiores implicadas en la competencia escrita muestra un cierto patrón evolutivo relacionado con el curso y/o el nivel de madurez de los alumnos, lo que a su vez parece derivar o tener efectos positivos en el producto escrito, evidenciándose productos textuales de mayor calidad en el alumnado mayor.

Estas conclusiones pueden discutirse del siguiente modo. Con respecto al producto textual, los resultados del presente estudio se secundan con investigaciones previas que muestran que cuanto mayor es la edad de los sujetos, más automatizada tienen la tarea escritora, especialmente los aspectos mecánicos, pudiendo focalizar su atención en la organización de ideas y dotar al texto de mayor calidad (Rodríguez y Gallego, 2017). Con respecto al proceso de planificación, las conclusiones del trabajo denotan cierta evolución en la adquisición de ciertas habilidades planificadoras (Limpo et al., 2014). En relación a la revisión, el hecho de que los alumnos de $4^{\circ}$ detectaran fundamentalmente errores de tipo mecánico se puede deber a que los alumnos de $4^{\circ}$ aun no poseen la suficiente madurez en la conceptualización de la escritura y por ello focalizan sus esfuerzos y prestan más atención a los procesos de bajo nivel cognitivo, considerando que un texto será de calidad si este carece de errores mecánicos (Limpo et al., 2014). Por su parte, la falta de diferencias entre grupos en relación a los errores sustantivos podría deberse a la complejidad de este tipo de errores y la demanda cognitiva que implica su detección. Es necesario haber automatizado previamente los aspectos mecánicos para poder regular los esfuerzos cognitivos y orientarlos hacia la detección de errores sustantivos (Chanquoy, 2001). En cuanto a las estrategias de corrección de errores, el mayor uso de estrategias de añadir y quitar contenido ante errores mecánicos puede deberse a que conllevan una menor carga cognitiva y han sido las primeras en adquirirse y asimilarse (Arias-Gundíny García, 2008). Por uso parte, la variedad en el uso de estrategias diversas para corregir errores sustantivos puede deberse, como se ha mencionado anteriormente, a la adquisición y asimilación gradual de las estrategias, de modo que una vez aprendidas las estrategias de añadir y quitar, se puede focalizar la atención de los procesos de revisión en otras estrategias que demandan más esfuerzos cognitivos (Arias-Gundín y García, 2008).

Finalmente, hay que señalar que las conclusiones del presente estudio deben considerarse en el contexto de las limitaciones del mismo, las cuales se presentan a continuación. Una de las principales limitaciones de la investigación desarrollada es que la muestra del estudio está muy localizada y limita la posibilidad de generalizar los resultados al resto de la población. Si bien, esta limitación podría suponer una futura prospectiva del estudio al plantear la ampliación del estudio a muestras más amplias, pertenecientes incluso a diferentes países de habla hispana, para comprobar de este modo si el proceso de aprendizaje y adquisición de las habilidades de escritura es similar en otros contextos hispanos. Otra limitación que presenta el estudio es 
la ausencia de un segundo corrector que corrigiera las tareas evaluativas, de modo que no se pudo llegar a un acuerdo inter-jueces que permitiera evitar la posible subjetividad en algunas medidas evaluativas, si bien la mayoría de las medidas consideradas eran de carácter objetivo. En este caso, se propone que, para futuras investigaciones se empleen otras medidas de carácter on-line que permitan obtener un registro más directo del proceso real de escritura y cuyo análisis arroje datos más precisos sobre la activación secuencial de los procesos escritores desplegados por los alumnos durante la tarea de composición escrita.

Pese a estas limitaciones, el estudio aporta información de interés científico al campo de estudio de la composición escrita en el contexto hispano hablante, pero además deriva en implicaciones de carácter práctico o aplicado al campo educativo propiamente dicho. En este caso, dado que la investigación, permite conocer cómo difieren las habilidades de planificación y revisión textual de los alumnos a medida que evolucionan en su escolarización primara se podría elaborar un programa o diseño curricular del proceso de enseñanza de la composición escrita adaptado a cada curso, en el que el docente instruya en aquellas habilidades escritoras que están en proceso de desarrollo y/o adquisición por parte de sus alumnos, contribuyendo con ello a su dominio y en consecuencia a la mejora de la calidad de los textos escritos de los estudiantes. Por otra parte, al conocer la evolución de estos procesos se podría mejorar el rendimiento de los alumnos en tareas de composición escrita y prevenir posibles dificultades en el aprendizaje de la escritura, abordando el tratamiento temprano de aquellos aspectos en los que los alumnos presenten mayores dificultades.

\section{REFERENCIAS}

Aguirre, L. A. y Negri, S. A. (2019). Proceso de revisión y calidad del producto textual. En D, Riestra. y N, Múgica. (Eds). Estudios SAEL 2019 (pp. 183-196). Argentina: Editorial de la Universidad Nacional del Sur. Disponible en http://hdl.handle.net/11336/130100

Álvarez-Fernández, M. L. y García-Sánchez, J. N. (2014). Evolución del proceso escritor desde la Educación Primaria a la Secundaria. Revista de Psicodidáctica, 19(1), 5-26. Disponible en http://hdl.handle.net/10810/48305

Arias-Gundín, O. y García, J. N. (2008). Adquisición de las habilidades implicadas en el proceso de revisión textual: estudio evolutivo en estudiantes de $5^{\circ}$ de Educación Primaria a $2^{\circ}$ de Educación Secundaria Obligatoria. International Journal of Developmental and Educational Psychology, 4(1), 435-443. Disponible en https://revista.infad.eu/index.php/ IJODAEP/issue/archive/2

Arias-Gundín, O. y García, J. N. (2007). La tarea de reescritura para evaluar la revisión textual. Boletín de Psicología, 90, 33-58. Recuperado de https:/www.uv.es/seoane/boletin/ previos/N90-3.pdf

Arias-Gundín, O. y García-Sánchez, J. N. (2006). El papel de la revisión en los modelos de escritura. Aula abierta, 88, 37-52. Recuperado de https://reunido.uniovi.es/index.php/AA/ issue/view/1034/134

Berninger, V. W. \& Swanson, H. L. (1994). Modifying Hayes and Flower's model of skilled writing to explain beginning and developing writing. In: E. C. Butterfield (Ed.), Children's writing: Toward a process theory of the development of skilled writing, (pp. 57-81). Greenwich: JAI Press. 
Chanquoy, L. (2001). How to make it easier for children to revise their writing: A study of text revision from 3rd to 5th grades. British Journal of educational psychology, 71, 15-41. https://doi.org/10.1348/000709901158370

Finney, S.J. \& DiStefano, C. (2006). Non-normal and categorical data in structural equation modeling. In: G. R. Hancock \& R. O. Muller (Eds.), Structural equation modeling: A second course (pp. 269-314). Greenwich, CT: Information Age.

García, M. (2015). Estrategias de aprendizaje para la adquisición de la competencia lingüística en alumnos de FLE. Anales de Filología Francesa, 23, 125-143. Recuperado de http://revistas.um.es/analesff/article/view/250611/190201

García, J. N. y Arias-Gundín, O. (2004). Intervención en estrategias de revisión del mensaje escrito. Psicothema, 16(2), 194-202. Recuperado de https:/www.unioviedo.es/reunido/ index.php/PST/article/view/8234/8098

González, E. A. (2020). La revisión en escritura colaborativa a través de un wiki: un estudio de caso. Lenguaje, 48(2), 328-353. https://doi.org/10.25100/lenguaje.v48i2.8804

Graham, S. (2018a). A revised writer (s)-within-community model of writing. Educational Psychologist, 53(4), 258-279. https://doi.org/10.1080/00461520.2018.1481406

Graham, S. (2018b). Introduction to Conceptualizing Writing. Educational Psychologist, 53(4), 217-219. https://doi.org/10.1080/00461520.2018.1514303

Hayes, J. R. (1996). A new framework for understanding cognition and affect in writing. In: M. Levy \& S. Ransdell (Eds.), The science of writing: theories, methods, individual differences and applications (pp. 1-27). Mahwah: Lawrence Erlbaun Associates.

Hayes, J. \& Flower, L. (1980). Identifying the organization of writing processes. En L.W. Gregg, E.R. Steinberg (Eds.), Cognitive processes in writing (pp. 3-30). Nova Jersey: Erlbaum.

Herrera, J. (2020). Evaluación de la calidad en la educación básica y media en Colombia. Cultura, Educación y Sociedad, 11(2), 125-144. http://dx.doi.org/10.17981/cultedusoc.11.2.2020.08.

Hurtado, S. (2019). Estudio exploratorio de las técnicas y hábitos de composición textual en el nivel universitario. Revista Lengua y Habla, 23, 15-29. Disponible en http://erevistas. saber.ula.ve/index.php/lenguayhabla/article/view/15660

Limpo, T., Alves, R.\& Fidalgo, R. (2014). Chindren's high-level writing skills: Development of planning and revising and their contribution to writing quality. British Journald of EducationalPsychology, 84(2), 177-193. https://doi.org/10.1111/bjep.12020

Reino de España. (9 de diciembre de 2013). Ley orgánica para la Mejora de la Calidad Educativa. [Ley Orgánica 8/2013]. Boletin Oficial del Estado: 295. Disponible en https://www. boe.es/buscar/doc.php?id=BOE-A-2013-12886

Reino de España. (28 de febrero de 2014). Por el que se establece el currículo básico de la Educación Primaria [Real Decreto 126/2014]. BOE: 52. Recuperado de https://www.boe. es/buscar/pdf/2014/BOE-A-2014-2222-consolidado.pdf 
Rodríguez, A. y Gallego, J. L. (2017). Aprendiendo a escribir durante la enseñanza obligatoria, ¿deseo o realidad? Calidoscopio, 15(1), 106-125. Disponible en http://revistas.unisinos.br/index.php/calidoscopio/article/view/cld.2017.151.09

Robledo-Ramón, P. \& García-Sánchez, J. N. (2013). Strategy instruction for writing composition at school and home. Estudios de Psicología, 34(2), 161-174. https://doi. org/10.1174/021093913806751438

Sarmiento, C. y Ojeda, L. (2018). Fortalecimiento de los procesos de lectoescritura a través de la implementación de estrategias pedagógicas apoyadas en las TIC. Cultura. Educación y Sociedad, 9(3), 945-950. http://dx.doi.org/10.17981/cultedusoc.9.3.2018.112

SPSS. (versión 24.0). Statistical Package for the Social Sciences (SPSS) [Software]. Nueva York: IBM. Disponible en https://www.ibm.com/analytics/spss-statistics-software

Torrance, M., Thomas, G. V. \& Robinson, E. J. (1999). Individual differences in the writing behaviour of undergraduate students. British Journal of Educational Psychology, 69, 189-199. https://doi.org/10.1348/000709999157662

Turgut, G. (2019). A Revision Model in Writing for Novice Writers with a Focus on Audience and Feedback. Journal of Contemporary Educational Research, 3(1), 6-19. http://dx.doi. org/10.26689/jcer.v3i1.439

Vásquez-Bustamante, O. (2018). Competencias lectoras y escritoras a través de la investigación como estrategia pedagógica. Cultura. Educación y Sociedad, 9(3), 9-18. http://dx.doi. org/10.17981/cultedusoc.9.3.2018.01

Victoria, A., Esquivel-Ancona, M. F. y Aldrete-Cortez, V. A. (2019). Prerrequisitos para el aprendizaje académico en niños con alto y bajo rendimiento escolar. Revista de Psicología y Ciencias del Comportamiento de la Unidad Académica de Ciencias Jurídicas y Sociales, 10(1), 6-17. https://oi.org/10.29059/rpcc.20190602-77

Vieiro, P. (2007). Psicopedagogía de la escritura. Madrid: Pirámide.

Patricia Robledo Ramón. Doctora en Psicología y Ciencias de la Educación. Premio extraordinario de Doctorado y Licenciatura. Lleva a cabo su actividad docente en los diferentes títulos de Grado, Máster y Doctorado de la Facultad de Educación de la ULE (España). Forma parte de grupo de Innovación Docente en la Enseñanza y el Aprendizaje (IDEA), participando en más de diez proyectos de innovación. Ha desempeñado cargos de gestión académica relacionados con la acción tutorial, la coordinación de cursos o la pertenencia a comisiones académicas. Su principal línea de investigación científica, dentro del grupo de investigación Cognición y Aprendizaje de la Escritura y la Lectura (CAEL), se centra en el papel de la familia en el desarrollo de la competencia lectoescritora del alumnado de Educación Infantil y Primaria. https://orcid.org/0000-0001-5623-3776

Vanesa García Gutiérrez. Maestra de Educación Infantil, con un Máster Universitario en Investigación en Psicología Educativa y Ciencias de la Educación, y diversos cursos de formación en Educación Infantil y Primaria. Actualmente inscrita en el programa de doctorado de Psicología Educativa y Ciencias de la Educación de la Universidad de León (España). Forma parte del grupo de Investigación de Cognición y Aprendizaje de la Lectura y la Escritura (CAEL) de la Universidad de León. https://orcid.org/0000-0001-8749-6878 